\title{
MATCHING PURSUITS LOW BIT RATE VIDEO CODING WITH CODEBOOKS ADAPTATION
}

\author{
Chien-Kai Peng†, Wen-Liang Hwang \\ †Electrical Engineering Department, National Tsing Hua University \\ łInstitute of Information Science, Academia Sinica, Taiwan
}

\begin{abstract}
We propose a codebook adaptation algorithm for matching pursuit low bit rate video compression. Matching pursuit dictionary is a set of basis functions. In practice, a function is approximated as a vector. According to the incoming data, the basis functions are modified similar to the minimum-point-finding algorithm in stochastic regression. Although matching pursuit low bit rate compressions have been studied intensively, bases adaptation in the dictionary is a new approach. We demonstrate the performance of our adaptation method on MPEG-4 video sequences.
\end{abstract}

\section{INTRODUCTION}

Recently, there is an increasing demand for video communications using low bit rate channels (with bit rate ranging from $10 \mathrm{kbit} / \mathrm{s}$ to $60 \mathrm{kbit} / \mathrm{s}$ ). Examples are wireless channel or video database access. Current DCT-based motion compensated transform, such as H.263 and MPEG-2 have difficulties when operated at bit rate below $20 \mathrm{kbit} / \mathrm{s}$. Many studied showed that when the motion residuals (prediction errors due to motion compensation) are coded at low-bit rates, the choice of the basis functions is extremely important. If a basis function can match the inner structures of a residual frame, the very limited bit budget can be used more efficiently in encoding the important structures in the frame, as a consequence, improving the coding performance[1][2][5]. In our previous research, we have trained the basis functions, called dictionary in matching pursuit, using techniques from Shape-Gain vector quantizer(VQ). The resultant basis set is then used to encode video sequences. Experiments indicated that this approach improved the PSNR performance in low-bit rate when the testing and training sequences contain slow-moving similar objects. However, for the sequences with different objects, basis functions optimized for one sequence may not be suitable to encode the other sequences. This says that for efficient low-bit rate video coding, the basis functions can not be fixed.
They depend on the video sequences and should be different from one sequence to the other sequence. This motivates our approach proposed in this paper: on-line adaptation of the basis functions for matching pursuit video codec.

In our approach, we adopt the Keifer-Wolfowitz techniques in stochastic regression, to modify the basis functions[7]. The Keifer-Wolfowitz is a solution to find the minimum-point-finding problem of a regression function. In [6], the optimized basis functions were obtained by the techniques from shape-gain VQ. The code books are obtained when the averaged distortion of the motion residual is a minimum. The adaptation algorithm deals with the scenario when the code books optimized for one sequence is modified on line for the other sequence. One can show that once the new sequence has infinite number of frames, the code books will converge to the optimized code book of the sequence. Experiments showed that with the code book adaptation, the PSNR performance as well as visual quality for video-sequence with slow-moving objects can be improved. The improvement increases as the number of the frame increases. The performance of the adaptation algorithm is not improved when the sequence contains fast-moving objects. For sequences with fast-moving objects, the motion prediction failed in many places. With a limited small amount of budget, purely from selection of the basis functions, the error structure can not be well-recoved. Although the adaptation algorithm improves the PSNR performance, there is a cost to be paid for the decoder: Since the basis functions are modified on line, the decoder side has to run a similar codebooks adaptation algorithm to keep up with the current basis functions.

In Section 2, we will review the matching pursuit theory. The use of the shape-gain VQ techniques in matching pursuit codec will be discussed in Section 3 . The adaptation algorithm will be proposed in Section 4. Experimental results are shown in Section 5. Final Section is the conclusion. 


\section{MATCHING PURSUIT THEORY}

For simplicity, we discussed only the one-dimensional matching pursuit algorithm[3]. One can extend easily the one-dimensional algorithm to two-dimensional by tensor product. Assume we have a large set of basis $D$ which spans densely all the $L^{2}(R)$ functions. Then an $L^{2}(R)$ function is representable with the following iterative procedure:

Let $g_{\gamma_{0}} \in D$. The vector $f$ can be decomposed into

$$
f=\left\langle f, g_{\gamma_{0}}\right\rangle g_{\gamma_{0}}+R f
$$

where $R f$ is the residual vector after approximating $f$ in the direction of $g_{\gamma_{0}}$. The matching pursuit algorithm then decomposes the residue $R f$ by projecting it on a vector of $D$ that matches $R f$ at best, as it was done for $f$. This procedure is repeated each time on the following residue that is obtained. They showed that when the dictionary is complete; then

$$
f=\sum_{n=0}^{+\infty}\left\langle R^{n} f, g_{\gamma_{n}}\right) g_{\gamma_{n}}
$$

The most common basis of a matching pursuit dictionary is a general family of time-frequency atoms and can be generated by scaling, translating and modulating a single function $g(x)$ with the following form:

$$
g_{\gamma}(t)=\frac{1}{\sqrt{s}} g\left(\frac{t-u}{s}\right) e^{i \xi}
$$

where we impose that $\|g(t)\|=1$ and scale $s>0$, frequency modulating $\xi$, translation $u$, and $\gamma=(s, u, \xi)$. In [1], the tensor product of an efficient subset of Eq. (1) was adopted as dictionary.

\section{DICTIONARY OPTIMIZATION WITH SHAPE-GAIN VQ}

In the VQ design, we quantize a random variable $X$. Our random variable is the collection of the image patches in the matching pursuit residual function $R^{n} f(t)$ where inner structure of the residual image can be well-described. In our implementation, each residual function provides one image patch; That is, if $N$ atoms are encoded for a residual frame; then there are $N$ image patches obtained from $R^{n} f(t)$ with $n=1, \cdots, N$. The image patch is approximately the block where the energy is the largest in $R^{n} f(t)$. Now, we will explain our method of employing gain-shape VQ technique to optimize the matching pursuit dictionary. In our gain-shape VQ, we have three codebooks[5][6]:

- Gain codebook : $C_{g}=\left\{\hat{g}_{i} ; i=1,2, \cdots, N_{g}\right\}$.
- $x$-axis shape codebook :

$$
C_{s x}=\left\{\hat{S} x_{j} ; j=1,2, \cdots, N_{S}\right\} .
$$

- The $y$-axis shape codebook :

$$
C_{s y}=\left\{\hat{S} y_{k} ; k=1,2, \cdots, N_{S}\right\} \text {. }
$$

There are four necessary conditions for the optimal codebook design:

1. Optimal partition for fixed $C_{g}, C_{s x}$, and $C_{s y}$,

2. Optimal $C_{g}$ for fixed $C_{S x}, C_{S y}$, and partition,

3. Optimal $C_{S x}$ for fixed $C_{S y}, C_{g}$ and partition,

4. Optimal $C_{S y}$ for fixed $C_{S x}, C_{g}$ and partition.

At the optimization steps from (2)-(4), we re-calculate the corresponding centroid at each partition. We will not give the detailed derivation of the centroid calculations in this summary. To find the optimize gain-shape matching pursuit dictionary, we will start from an initial basis and iterative along all the necessary conditions until some criterion is matched. A good initial subset of the dictionary can be obtained from Neff and Zakhor's method[1]: The motion residual image to be coded by matching pursuit is first divided into blocks, and the sum of the squares of all pixel intensities is computed for each block. The center of the block with the largest energy value is selected as an initial estimate for the inner product search. The procedure is iteratived several times. A subset of the dictionary that occurs frequently for the training sequences are then selected.

\section{MATCHING PURSUIT WITH CODEBOOKS ADAPTATION}

\subsection{System Description}

The motion model used by the matching pursuit system is identical to the advanced prediction model used by TMN[4], which is a specific implementation of H.263. The motion residual is coded by matching pursuits. Our system is similar to those used in [1][5] except that there is an adaptation algorithm both in encoder and decoder. In order to compare with H.263, the buffer regulation similar to in [1][5] is adopted. This regulation allows the matching pursuit system allocates a similar number of bits to each frame as that obtained by H.263. 


\subsection{Successive Parameter Estimation Method}

Our adaptation algorithm adopts the techniques in successive parameter estimation. In the success parameter estimation problem, the determination of the parameters is not from all the observed data, the parameters is updated from incoming data one by one and, hopefully with a sufficient number of data, the estimated parameters will converge to the "true" parameters. In our setting, the parameters to be updated are the code words, or the basis functions in current dictionary. There are two popular stochastic approximation methods for root-finding and minimum-point-finding, called Robbins-Monro and Kiefer-Wolfowitz methods, respectively. They are similar and we will review only 1-D case. The multidimensional extension of the methods can be found in [7].

Let $\theta$ and $\mathbf{z}$ be two random variables with some correlation, see Figure 1. A stochastic regression method is to find the root of the regression function $f(\theta)$, which is defined by

$$
f(\theta)=E\{\mathbf{z} \mid \theta\}
$$

In Robbins-Monro method, the new successive estimate $\theta_{N+1}$ based on the present estimate $\theta_{N}$ and a new observation $\mathbf{z}_{N}$ is given by

$$
\theta_{N+1}=\theta_{N}-a_{N} z_{N}
$$

where $a_{N}$ is a learning factor, which determines the updating codeword's convergence rate and assumed to be a sequence of positive numbers which satisfy the following conditions:

1. $\lim _{N \rightarrow \infty} a_{N}=0$,

2. $\sum_{N=1}^{\infty} a_{N}=\infty$,

3. $\sum_{N=1}^{\infty} a_{N}^{2}<\infty$.

The physical meaning of these equations are described as follows. The (1) allows the process to settle down in the limit, the (2) insures that there is enough corrective action to avoid stopping short of the root, and the (3) guarantees the variance of the noise to be finite so that the algorithm can correct the effect of the noise progressively. One can show that once a sequence $\left\{a_{N}\right\}$ satisfying the above 3 conditions, the adaptation method given in Eq.(3) converges to the root of Eq.(2) in the mean-square sense:

$$
\lim _{N \rightarrow \infty} E\left\{\left(\theta_{N}-\theta_{0}\right)^{2}\right\}=0,
$$

and

$$
\lim _{N \rightarrow \infty} P\left\{\theta_{N}=\theta_{0}\right\}=1
$$

The root-finding algorithm can be easily modified to seek the minimum point of a regression function: As is well known, the minimum point or the optimum point of a function is a root of the derivative of the function.

\subsection{CodeBooks Adaptation}

We will discuss our adaptation algorithm. Assume that the initial codebooks are given. The centroids of them are

$$
\left\{\hat{g}_{1}, . ., \hat{g}_{n}, \hat{s}_{1}, \ldots, \hat{s}_{m}\right\}
$$

where $\left\{\hat{g}_{i} \mid i=1, \ldots, n\right\}$ are centroids for gain codebook and $\left\{\hat{s}_{i} \mid i=1, . ., m\right\}$ are centroids for shape codebooks. Let $G_{i}$ be the union of the partitions with the $i$-th gain codeword, and $S_{i}$ be the union of the partitions with the $i$-th shape codeword, and $G$ is the union of $G_{i}, S$ is the union of $S_{i}$. An incoming realization $X$ falls to the partition with the $i$-th gain codeword and the $j$-th shape codeword has a distortion:

$$
d(X, G S)=\|X\|^{2}+\hat{g}_{i}^{2}-2 \hat{g}\left(X^{t} . \hat{s}_{j}\right) .
$$

This function is our regression function. The KieferWolfowitz method is used to minimize this distortion function. The partition with $i$-gain codeword and $j$ shape codeword will be updated because of this new incoming data $X$. The new gain centroid is :

$$
\hat{g}_{i}^{(n+1)}=\hat{g}_{i}^{(n)}-2 a_{n}\left(\hat{g}_{i}^{(n)}-X^{t} \hat{s}_{j}^{(n)}\right),
$$

where $n$ is the time-index and $a_{n}$ is the $n$-th term of a sequence satisfying (1)-(3) in previous subsection. In our adaptation algorithm, the gain centroids not in $G_{i}$ are preserved, that is, $\hat{g}_{k}^{(n+1)}=\hat{g}_{k}^{(n)}$, for $k$ not $i$.

The shape codebooks will be updated similarly like the gain codebook. We have $\mathrm{x}$-codebook and $\mathrm{y}$-codebook to be modified. We have shown that the adaptation of the $j$-th shape centroid in $\mathrm{x}$-codebook is :

$$
\hat{s}_{x j}^{(n+1)}=\hat{s}_{x j}^{(n)}+2 \hat{g}_{i} a_{n} \cdot X^{t} \cdot \hat{s}_{x j}^{(n)} .
$$

Then we normalize $\hat{s}_{x j}^{(n+1)}$ to 1 . Also, the $j$ th shape centroid in $\mathrm{y}$-codebook is modified:

$$
\hat{s}_{y j}^{(n+1)}=\hat{s}_{y j}^{(n)}+2 \hat{g}_{i} a_{n} \cdot X^{t} \cdot \hat{s}_{y j}^{(n)} .
$$

Like gain codebook, the centroids not in $S_{j}$ are preserved, that is, $\hat{s}_{x k}^{(n+1)}=\hat{s}_{x k}^{(n)}$, and $\hat{s}_{y k}^{(n+1)}=\hat{s}_{y k}^{(n)}$, for $k$ not $j$.

\section{EXPERIMENTAL RESULTS}

Our performance evaluations were done in conditions where the video sequences are coded at $24 \mathrm{kbit} / \mathrm{s}, 10$ 
frame/s, and with QCIF format. The comparisons were carried out with the basis function used in [1] as well that obtained by H. 263[4]. We began our adaptation with basis functions in [1]. Figures 2 and 3 show the PSNR for Akiyo and Hall Monitor sequences, respectively. One can see from these figures that our bases(marked by AMS) outperforms the others (marked by $\mathrm{H} .263$ and MP) when the frame number is large. In the beginning of the sequence, there is not sufficient number of frames from the new video sequence, as the frame number increases, a better estimation of the statistics corresponding to the new sequence leads to improving the PSNR performance.

\section{CONCLUSION}

We have proposed an apatation algorithm which modifies the bases functions of the matching pursuit dictionary on-line. Experiments show that method improves the PSNR performance when video sequences have slow-moving objects.

\section{REFERENCE}

[1] R. Neff and A. Zakhor, "Very low bit-rate video coding based on matching pursuits",IEEE Trans. Circuit and systems for video technology, 1997.

[2] M. R. Banham and J. C. Brailean, "A selective update approach to matching pursuits video coding", $I E E E$ Trans. Circuit and systems for Video Technology, 1997. [3] S. Mallat and Z. Zhang, "Matching Pursuits with time-frequency dictionaries",IEEE Trans. Signal Processing, 1993.

[4] "ITU Telecommunication Standardization Sector LBC95", Video Codec Test Model TMN 5.

[5] Y. T. Chou, W. L. Hwang, and C. L. Huang,"Very low-bit video coding based on gain-shape $\mathrm{VQ}$ and matching pursuits",IEEE ICIP, 1999.

[6] M. J. Sabin and R. M. Gray, "Product code vector quantizers for waveform and voice coding", IEEE Trans. on ASSP, 1984.

[7] K. Fukunaga,"Statistical Pattern Recognition",Academic Press, 1990.

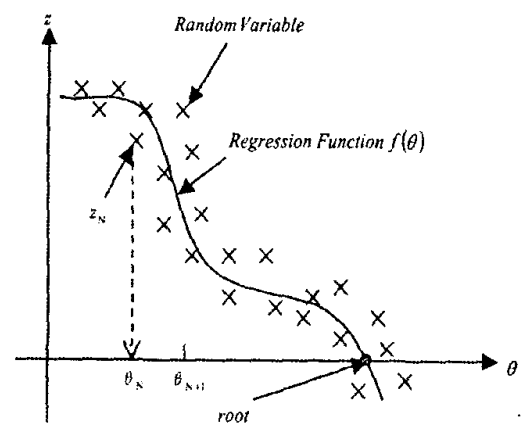

Figure 1: Root-Finding-Regression-Method

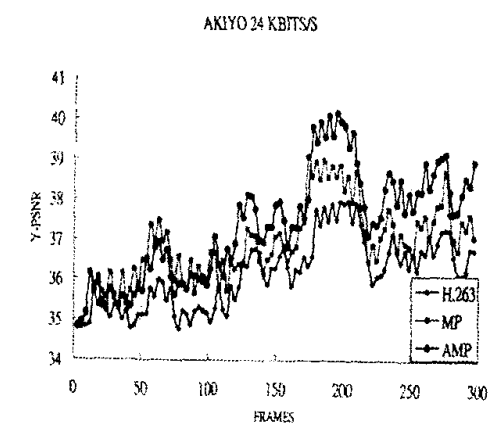

Figure 2: The PSNR comparison for Akiyo Sequence

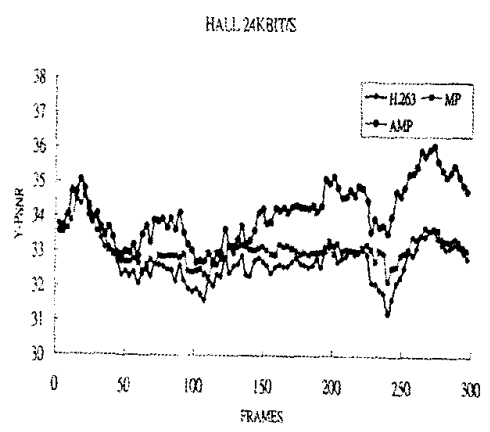

Figure 3: The PSNR comparison for Hall Monitor Sequence 